\title{
RELACIONES CLIMÁTICAS ENTRE LA FRACTALIDAD TEMPORAL DE LOS EPISODIOS DE LLUVIA Y DE SEQUÍA
}

\author{
Robert $\mathrm{MONJO}^{1 *}$, Javier MARTIN-VIDE ${ }^{2}$ \\ ${ }^{1}$ Fundación para la Investigación del Clima (FIC), C/ Gran Vía 22 (duplicado), 7º 28013 Madrid. \\ ${ }^{2}$ Grup de Climatologia, Departament de Geografia, Universitat de Barcelona, C/ Montalegre 6, \\ 08001 Barcelona. \\ *robert@ficlima.org
}

\section{RESUMEN}

La concentración de la lluvia y la recurrencia de las sequías suelen estudiarse de forma independiente. Sin embargo, el comportamiento fractal de la lluvia sugiere que existen relaciones de similitud a diferentes escalas temporales entre ambos aspectos climatológicos. En este trabajo se ha tomado un conjunto de 66409 series temporales de precipitación diaria de todo el mundo y se han obtenido diferentes patrones de semejanza entre la concentración de la lluvia y el comportamiento fractal de las rachas, tanto secas como húmedas. Usando índices de irregularidad tales como el Theil, Atkinson, Gini y el índice $n$, se han encontrado relaciones estadísticas altamente significativas entre las medidas de fractalidad, entropía y concentración. Este estudio podría servir para caracterizar los climas del mundo según la fractalidad de los episodios de lluvia. Además, las relaciones entre los diferentes índices permite optimizar la caracterización y medición de los cambios climáticos en los patrones de precipitación y sus impactos en la sociedad.

Palabras clave: concentración, fractalidad, precipitación diaria, sequía.

\begin{abstract}
Rainfall concentration and drought recurrence are usually studied independently. However, the fractal behavior of the precipitation suggests that there are similarity relations to different time scales between both climatic features. In this paper we have taken a set of 66,409 time series worldwide and have obtained different patterns of similarity between the rainfall concentration and the fractal behavior of both dry and wet spells. Using irregularity indices such as Theil, Atkinson, Gini and index $n$, highly significant statistical relationships were found between measures of fractality, entropy and concentration. This study could serve to characterize the climates of the world according to fractality of rainfall episodes. In addition, relations between different indexes will optimize the characterization and measurement of climate change in precipitation patterns and their impacts on society.
\end{abstract}

Key words: concentration, fractality, rain, precipitation, drought.

\section{INTRODUCCIÓN}

La intensidad media de la precipitación depende de la escala temporal considerada. La lluvia a escala diaria es fácilmente medible mediante pluviómetros tradiciona- 
les; sin embargo, la intensidad a escala sub-horaria presenta importantes dificultades para ser medida debido a su gran irregularidad (Liu et al. 2013, Monjo 2016). Una forma de medir la irregularidad de la precipitación es mediante índices asociados a la curva de Lorenz o la entropía estadística (Monjo y Martin-Vide, 2016). Por ejemplo, el Î́ndice de Concentración de Martín-Vide (2004) se relaciona climáticamente con el porcentaje de días de lluvia que acumulan la mayor parte de la precipitación anual, de acuerdo con la integral de la curva de Lorenz (Benhamrouche et al., 2015).

Por otro lado, existen algunos indicadores de la concentración temporal de la precipitación basados en la intensidad sub-diaria. Sin embargo, la mayoría de estos índices están diseñados a partir de umbrales específicos obtenidos para una determinada región climática. Un ejemplo es el índice $\beta$, diseñado para la región mediterránea (Llasat 2001). El índice $\beta$ de un evento de lluvia se define como la fracción de lluvia acumulada con una intensidad 5-minutal igual o mayor que $35 \mathrm{~mm} / \mathrm{h}$.

El índice IP de Casas et al. (2010) también clasifica la precipitación sub-diaria, de acuerdo con la relación entre las intensidades y duraciones (curvas de Intensidad-Duración-Frecuencia). En particular, el índice IP establece umbrales variables según el clima: Considera un período de retorno de 5 años y cuatro duraciones, que son 5 minutos, 1,2 y 24 horas.

El índice $n$ de Monjo (2016) se define como el exponente adimensional de las curvas Intensidad-Duración y puede interpretarse como la dimensión fractal de la intensidad de la lluvia. El índice $n$ presenta un umbral teórico que generalmente es independiente de la intensidad y la duración del evento. El umbral $(n=0,5)$ permite discriminar entre un predominio de lluvias regulares $(n<0,5)$ e irregulares $(n>0,5)$, de acuerdo con la distribución de la concentración de las intensidades de la lluvia dentro de un evento. Esto a su vez se relaciona con procesos (advección / convección) que intervienen en la formación de la precipitación.

Además de la intensidad, otros comportamientos de la precipitación pueden ser descritos mediante una dimensión fractal, obtenida por ejemplo usando el método del box-counting, o el exponente de Hurst (Feder 1988, Olsson 1992, Taouti y Chettih 2014, Meseguer-Ruiz et al 2016). Sin embargo, estos métodos analizan sólo algunas de las características de precipitación por separado, como son la persistencia o su intensidad, pero no analizan conjuntamente la relación entre la concentración de las rachas húmedas/secas y la concentración de los días de lluvia.

Este trabajo explora las relaciones matemáticas de la estructura fractal de las rachas húmedas y secas, conjuntamente con otras características climáticas tales como la concentración o la irregularidad de la lluvia diaria. Estas relaciones son fundamentales para caracterizar la variabilidad climática natural y con ello estimar posibles cambios que deriven de modificaciones en los patrones de circulación global.

\section{DATOS}

En total se usaron 66409 series temporales de precipitación diaria disponibles para el periodo 1950-2014, tomadas del Global Historical Climatology NetworkDaily Database (GHCN-Daily) (Menne et al., 2012). Las estaciones seleccionadas se corresponden con aquellas que presentan al menos 1000 valores diarios con al menos un 70\% de continuidad. De las 66409 estaciones, más de la mitad presentan más de 8000 registros, y más del 75\% superan los 3000 registros con un $98 \%$ de 
continuidad temporal. Aproximadamente el 25\% de las estaciones destacan por tener más de 20000 registros diarios con el $99 \%$ de continuidad.

Un estudio de la calidad de los datos fue llevado a cabo usando el test de Kolmogorov-Smirnov, prueba para detectar inhomogeneidades (Monjo et al., 2013). Sólo el 5\% de las series temporales presentaron algunas inhomogeneidades, y, en estos casos, se seleccionó la sub-serie temporal homogénea más larga.

En promedio, aproximadamente hay un observatorio por cada $244 \mathrm{~km}^{2}$ de la superficie terrestre. Sin embargo, la cobertura espacial es muy desigual dependiendo del país analizado. En particular, esto depende generalmente de su desarrollo económico o su antigua dependencia colonial (Fig. 1). Entre los países con mayor densidad de estaciones se incluyen los EE.UU., Brasil, India, Australia y los Países Bajos. África presenta una baja densidad en prácticamente toda su extensión, a excepción del sur.

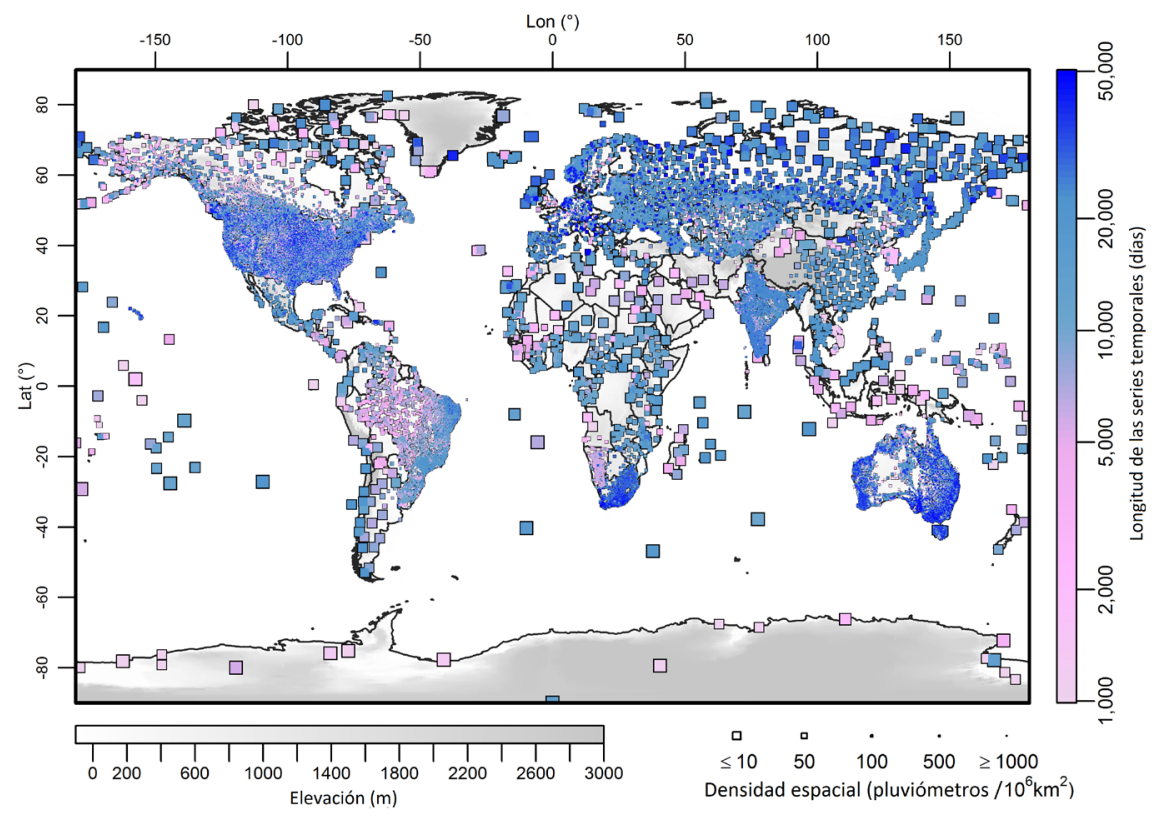

Fig 1. Ubicación de los pluviómetros que han suministrado las series temporales, tomados de la Global Historical Climatology Network-Daily Database. El color indica la duración de las series temporales (en días). El tamaño del punto es inversamente proporcional a la densidad de puntos en cada área.

\section{METODOLOGÍA}

\subsection{Medidas de la concentración, desigualdad e irregularidad}

La mayoría de los índices de concentración/desigualdad empleados en este trabajo se basan en la integral o la forma de la curva de Lorenz, que relaciona la acumulación de una variable $(y)$ y su frecuencia acumulada $(x)$. Por ejemplo, a partir de esta curva, se define el índice de Gini $(G I)$ : 


$$
G I=\frac{S}{S+A}=1-2 A
$$

donde $S$ es el área entre la curva $y(x)$ y la recta equidistributiva $y=x$. Por lo tanto, $S$ es igual a $1 / 2-A$, donde $A$ es el área debajo de la curva $y(x)$, que puede estimarse usando la regla del trapezoide.

En particular se tomaron los índices de Theil $(T I)$, Gini $(G I)$, Atkinson $(A I)$, Monjo $\left(n_{\delta}\right.$ y $\left.n_{\text {or }}\right)$ y la Desviación Media Logarítmica / Entropía Generalizada $(G E)$, que miden magnitudes muy relacionadas entre sí: concentración, desigualdad, entropía o irregularidad (Tabla 1).

\begin{tabular}{cccc}
\hline Índice & Magnitud & Cuva de & Referencia \\
\hline$G I$ & Concentración-desigualdad & Sí & Haughton y Khandker (2007) \\
$G E$ & Entropía-desigualdad & Sí & Monjo y Martin-Vide (2016) \\
$T I$ & Entropía-desigualdad & No & Jost (2006) \\
$A I$ & Desigualdad & Sí & Atkinson (1970) \\
$n_{\text {or }}$ & Concentración & Sí & Monjo y Martin-Vide (2016) \\
$n_{\delta}$ & Irregularity & No & Monjo (2016) \\
\hline
\end{tabular}

Tabla 1. Índices empleados y la magnitud que miden. En la tercera columna se indica si requieren una reordenación de los valores (curva de Lorenz) en vez de mantener la estructura de las series temporales. Para más detalles ver Monjo y Martin-Vide (2016)

\subsection{Medidas de la fractalidad}

La duración fractal de la lluvia puede medirse usando la dimensión del boxcounting o contaje de bloques (Taouti \& Chettih 2014), también conocido como dimensión de Minkowski-Bouligand, que a su vez está fuertemente relacionada con la dimensión de Hausdorff-Besicovitch (Falconer 2003).

A partir de una serie diaria de precipitación y agrupándola en bloques (sin solapamientos) de longitud $r$, puede definirse $N_{r}$ como el número de bloques con precipitación acumulada mayor que cero. La longitud total (en días) de los bloques húmedos vendrá dada por $D_{r}=r N_{r}$. Esta cantidad se conoce como duración fractal de la serie de precipitación. La relación entre la duración $D_{r}$ y la resolución $r$ viene dada por la fórmula:

$$
D_{r}=c \cdot r^{d}
$$

donde $d$ es la dimensión fractal y $c$ es la constante de duración. La resolución $r$ es la duración, en días, de cada bloque de tiempo. Para poder comparar entre observatorios, la duración $D_{r}$ es normalizada a la unidad, y por tanto también la constante $c$ queda expresada como un ratio (es decir, número de días húmedos dividido entre días totales). Nótese que la duración fractal de la lluvia está comprendida entre el caso instantáneo $(d=1)$ y el caso de lluvia constante $(d=0)$. La definición de duración fractal puede entenderse mejor en el ejemplo de la Tabla B1. 


\begin{tabular}{|c|c|c|c|c|c|c|c|c|c|c|c|c|c|c|}
\hline $\boldsymbol{r}$ & \multicolumn{14}{|c|}{$p_{j, r}$} \\
\hline 1 & 1 & 3 & 5 & 3 & 0 & 0 & 0 & 20 & 0 & 0 & \begin{tabular}{l|l}
0 & 0
\end{tabular} & \begin{tabular}{l|l}
0 & 0
\end{tabular} & 0 & 1 \\
\hline 2 & 4 & 4 & $\varepsilon$ & & c & ) & 2 & 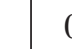 & 0 & 0 & & 0 & & 1 \\
\hline 4 & \multicolumn{4}{|c|}{12} & \multicolumn{4}{|c|}{2} & \multicolumn{2}{|c|}{0} & \multicolumn{4}{|c|}{1} \\
\hline 8 & \multicolumn{7}{|c|}{14} & \multicolumn{7}{|c|}{1} \\
\hline 16 & \multicolumn{14}{|c|}{15} \\
\hline
\end{tabular}

\begin{tabular}{ccccc}
$\boldsymbol{N}_{\boldsymbol{r}}$ & $\boldsymbol{r} \boldsymbol{N}_{\boldsymbol{r}}$ & $\begin{array}{c}\boldsymbol{r} \cdot \boldsymbol{N}_{\boldsymbol{r}} \\
\boldsymbol{I 1 6}\end{array}$ & $\boldsymbol{D}_{\boldsymbol{r}}$ & $\boldsymbol{a}_{\boldsymbol{r}}$ \\
\hline 6 & 6 & 0,38 & 0,38 & 2.50 \\
4 & 8 & 0,5 & 0,47 & 1.88 \\
3 & 12 & 0,75 & 0,69 & 1.25 \\
2 & 16 & 1 & 0,75 & 0,94 \\
1 & 16 & 1 & 1 & 0,94 \\
\hline
\end{tabular}

Tabla 2. Ejemplo de una serie pluviométrica de 16 días. Valores de precipitación $p_{j, r}(\mathrm{en} \mathrm{mm})$ dependiendo de la resolución $r$ (en días). Las otras cantidades son: el número de cuentas con lluvia $N_{r}$, la duración fractal $D_{r}$ (Ec. 2) y la intensidad media $a_{r}(E c$. 3) en mm/dia.

Dada una determinada resolución temporal $r$, la intensidad media $a_{i r}$ de cada racha " $i$ " de lluvia se define como el cociente entre la acumulación total $P_{i r}$ y la duración de la racha $D_{i r}$. Así, se define la intensidad media $a_{r}$ de una serie temporal de precipitación como la media de todas las intensidades $a_{i r}$. Estos valores se relacionan con la resolución según:

$$
\frac{a_{r}}{a_{1}}=\left(\frac{r_{1}}{r}\right)^{m}
$$

donde $m$ es la dimensión de la precipitación media.

Análogamente al índice $n_{\delta}$ (Tabla 1), puede calcularse una dimensión fractal de las rachas húmedas y secas. Para ello basta con utilizar la duración $D_{i}$ (con resolución $r=1$ ) como si fuera un valor de precipitación de un evento, y tomar el umbral $D_{i}=1$ como valor "seco". Por ejemplo, la serie de rachas secas o húmedas $D_{i}=(3,4,7,1,3$, $10,12)$ se transforma en dos "eventos" independientes $\{(3,4,7),(3,10,12)\}$

Para cada evento de rachas, se define la duración media máxima $Y_{i}$ como una variable dentro de la racha, de acuerdo con:

$$
Y_{i}=\frac{1}{i} \max _{n-i+1}\left\{\sum_{j=k}^{k+i-1} D_{j}\right\}_{k=1}^{n-i+1}
$$

Por lo tanto, la duración media máxima satisfará también una relación del tipo:

$$
\frac{Y_{i}}{Y_{1}}=\left(\frac{1}{i}\right)^{h}
$$

Se denota con $h$ al exponente, o la dimensión fractal, de las rachas húmedas. Mientras que se usa $s$ para la dimensión fractal de las rachas secas. En definitiva, los índices que nos interesan son: $c, d, m, h$ y $s$. 


\section{RESULTADOS Y DISCUSIÓN}

\subsection{Relaciones específicas de concentración-desigualdad}

El primer resultado que llama la atención es la elevada correlación, estadísticamente significativa, que existe entre la mayoría de los índices analizados. Por ejemplo, los índices de concentración, desigualdad e irregularidad se relacionan entre sí de forma directa, mientras que la entropía $G E$ presenta una relación inversa (Tabla 2). Cabe resaltar que la mayoría de esas propiedades pueden resumirse mediante el índice de Gini $(G I)$, ya que presenta la mayor correlación con todos los demás, y en todos los casos es elevada $(\mathrm{R}>0,7)$ y significativa $(p$-valor $<0,001)$.

\begin{tabular}{|c|c|c|c|c|c|c|}
\hline Comparando & $G I$ & $n_{o r}$ & $n_{\delta}$ & $T I$ & $A I$ & $-G E$ \\
\hline$G I$ & - & 0,98 & 0,71 & 0,76 & 0,91 & 0,71 \\
\hline$n_{o r}$ & 0,98 & - & 0,63 & 0,76 & 0,81 & 0,65 \\
\hline$n_{\delta}$ & 0,71 & 0,63 & - & 0,55 & 0,71 & 0,63 \\
\hline$T I$ & 0,75 & 0,76 & 0,55 & - & 0,61 & 0,92 \\
\hline$A I$ & 0,91 & 0,81 & 0,71 & 0,61 & - & 0,64 \\
\hline$-G E$ & 0,71 & 0,65 & 0,63 & 0,92 & 0,64 & - \\
\hline Media & 0,81 & 0,77 & 0,65 & 0,72 & 0,74 & 0,71 \\
\hline
\end{tabular}

Tabla 2. Correlación de Pearson de los índices de concentración-desigualdad-irregularidad. Todas las relaciones son estadísticamente significativas ( $p$-valor $<0,001)$.

\subsection{Relaciones entre concentración-desigualdad y fractalidad}

Existe una relación débil pero estadísticamente significativa entre el índice de estructura de Monjo $\left(n_{\delta}\right)$ y algunos índices de fractalidad tales como la dimensión de la duración $d(R=0,22, p$-valor $<0,001)$, la dimensión de las rachas húmedas $h(R=-0,21$, $p$-valor $<0,001)$ y la de las rachas secas $s(R=-0,19, p$-valor $<0,001)$. El primer caso puede ser deducido teóricamente de acuerdo con la interpretación fractal del índice $n$ (Monjo, 2016). Por otro lado, la correlación negativa con $h$ y $s$ implica que una mayor concentración de las rachas está asociada a una mayor regularidad de las precipitaciones. Por ejemplo, si predominan las rachas húmedas y de gran duración seguidas de rachas muy cortas (es decir, $h>0,5$ ), entonces cabe esperar que exista una gran persistencia de la lluvia dentro de esas rachas $(n<0,5)$. Por lo contrario, si todas las rachas son cortas y por tanto de duración similar $(h<0,5)$, suele estar ligado a una mayor irregularidad de la lluvia $(n>0,5)$.

Las relaciones entre el índice de concentración $(G I)$ y las medidas de fractalidad también son en general débiles, aunque son significativas para la constante de duración $c(\mathrm{R}=0,20, p$-valor $<0,001)$, la dimensión de la precipitación media $m,(\mathrm{R}=0,22$, $p$-valor $<0,001)$ y la dimensión de las rachas húmedas $h(\mathrm{R}=0,19, p$-valor $<0,001)$. Estas conexiones se explican porque la variabilidad diaria de la lluvia $(G I>0)$ se refleja en la variabilidad de la precipitación media $(m>0,5)$ y de las rachas $(h>0,5)$. Por su parte, el índice de Atkinson (AI) presenta correlaciones significativas con $d, h$, $c$ y $m$, siendo $\mathrm{R}=-0,22$ para $d \mathrm{y}$ en torno a $\mathrm{R}=0,23$ para el resto. La interpretación de 
este resultado es similar a los casos anteriores, con la salvedad de que $A I$ se relaciones de forma inversa con la duración fractal de la lluvia $(d)$.

\subsection{Relaciones específicas entre los índices de fractalidad}

Las conexiones entre las medidas de fractalidad son incluso mayores que en muchos de los casos anteriores (Fig. 2). La dimensión de las rachas húmedas $(h)$ está directamente relacionada con la dimensión de la intensidad media $(m)$ y con la duración media $(c)$. Es decir, cuanto mayor es la duración media de las rachas, mayor es el número de casos de rachas largas rodeadas de rachas cortas. Más sorprendente es la relación directa entre $m$ y $h$ : Se produce una mayor concentración media de la lluvia cuando se producen rachas largas rodeadas de cortas.
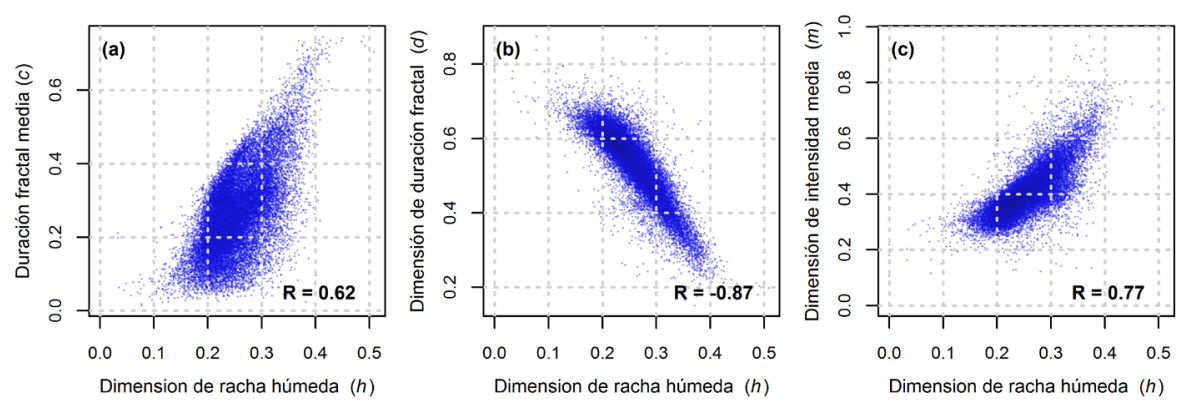

Fig 2. Relaciones entre la dimensión de las rachas húmedas y otros indices fractales: (a) duración media, (b) dimensión de la duración y (c) dimensión de la duración media. Las correlaciones $(R)$ presentan una significación del p-valor $<0,001$.
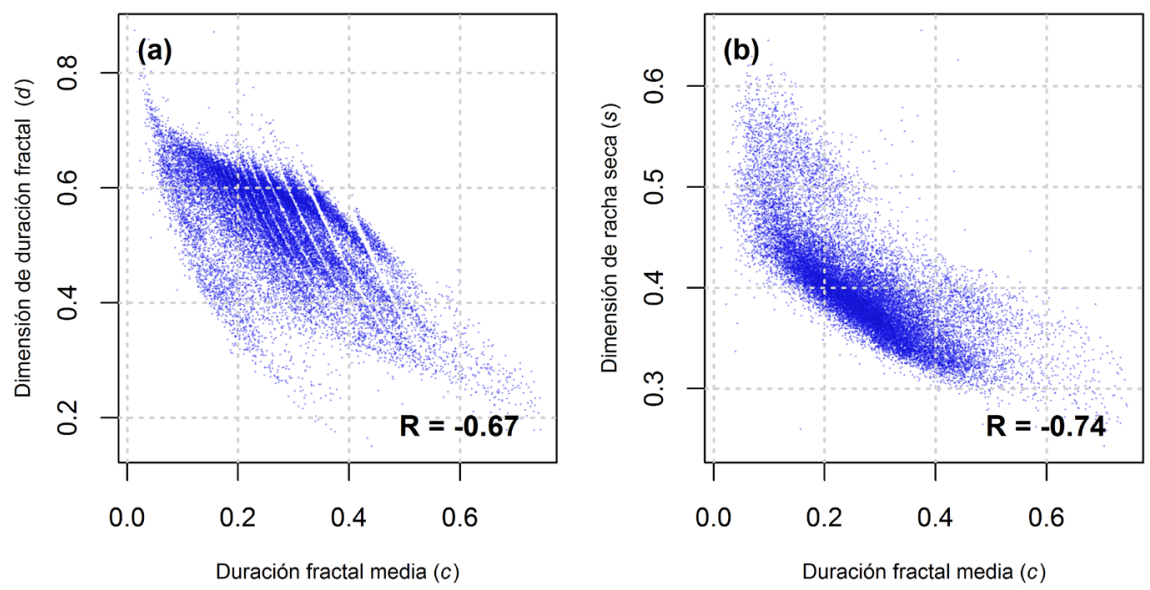

Fig 3. Relación entre la duración fractal media con (a) la dimensión de la duración y (b) dimensión de la racha seca. Las correlaciones $(R)$ presentan una significación del p-valor $<0,001$.

Además, se encontró una relación inversa entre la dimensión de la racha húmeda y su duración (Fig. 2b). Es decir, el mayor contraste de rachas húmedas $(h>0,5)$ pro- 
voca que éstas tiendan a ser persistentes $(d<0,5)$, tal como se desprende de los bajos valores del índice $n$, según el apartado 4.2.

La duración media de las rachas también se relaciona de forma inversa con su dimensión (Fig. 3a) y con la dimensión de la racha seca (Fig. 3b), al contrario que con la dimensión de la racha húmeda (Fig. 2a). Por lo tanto, puede decirse que a mayor duración de las precipitaciones $(c>0,4)$ en general se produce una mayor constancia de las mismas.

\section{CONCLUSIONES}

Se encontraron fuertes conexiones entre los índices analizados, y por lo tanto pueden seleccionarse unos pocos para representar la mayoría de las características estudiadas. Por ejemplo, para las propiedades de concentración de la lluvia, se recomienda utilizar el índice de Gini $(G I)$ debido a su simple estimación y su elevada correlación $(R>0,7)$ con la mayoría de los índices de irregularidad y entropía.

Por otro lado, este estudio revela que la precipitación generalmente tiene una estructura anidada en el tiempo. Es decir, existe una relación de auto-similitud en varias escalas temporales, de tal modo que cada acumulación máxima de duración más corta se incluye dentro de las más largas. En este sentido, el índice $n$ puede ser interpretado como una dimensión fractal de las intensidades medias máximas, fuertemente relacionado con las dimensiones fractales de la lluvia: duración, intensidad media y estructura temporal de las rachas húmedas y secas.

Finalmente, de las conexiones entre los diferentes índices de fractalidad se desprende que una mayor duración de las precipitaciones $(c>0,4)$ generalmente implica una mayor constancia de las mismas $(d<0,5)$, así como también se relaciona con una mayor homogeneidad en la distribución temporal de las precipitaciones $(n<0,5)$.

Este estudio podría servir para caracterizar la variabilidad climática a escala mundial según diferentes medidas de fractalidad y concentración de las precipitaciones. Además, los índices analizados resultan útiles para la detección y medición de las variaciones que podrían sufrir los patrones de precipitación en el contexto del cambio climático y su influencia en la erosión.

\section{REFERENCIAS}

Benhamrouche, A.; Boucherf , D.; Hamadache, R.; Bendahmane, L.; MartinVide, J.; Teixeira Nery, J. (2015). Spatial distribution of the daily precipitation concentration index in Algeria. Nat Hazards Earth Syst Sci, 15, 617-625. doi: http://dx.doi.org/10,5194/nhess-15-617-2015

Casas, M.C.; Rodríguez, R.; Redaño, Á. (2010). Analysis of extreme rainfall in Barcelona using a microscale rain gauge network. Meteorological Applications 17, 117-123 doi: http://dx.doi.org/10,1002/met.166

Feder. J. (1988). Fractals. Plenum press, New York.

Llasat, M.C. (2001). An objective classification of rainfall events on the basis of their convective features. Application to rainfall intensity in the North-East of Spain. Int J Climatol 21, 1385-1400, 
Liu, X.C.; Gao, T.C.; Liu, L. (2013). A comparison of rainfall measurements from multiple instruments. Atmos Meas Tech 6, 1585-1595. doi: http://dx.doi. org/10,5194/amt-6-1585-2013

Martín-Vide, J. (2004). Spatial distribution of a daily precipitation concentration index in peninsular Spain. Int J Climatol 24, 959-971.

Menne, M.J.; Durre, I.; Vose, R.S.; Gleason, B.E.; Houston, T.G. (2012). An Overview of the Global Historical Climatology Network-Daily database. J Atmos Ocean Tech 290, 897-910, Data: ftp://ftp.ncdc.noaa.gov/pub/data/ghcn/daily/ (accedido 10 Junio 2016).

Meseguer, Ó.; Olcina, J.; Sarricolea, P.; Martin-Vide, J. (2016): The temporal fractality of precipitation in mainland Spain and the Balearic Islands and its relation to other precipitation variability indices. Int. J. Climatol. doi: 10.1002/joc.4744.

Monjo, R.; Pórtoles, J.; Ribalaygua, J. (2013). Detection of inhomogeneities in daily data: a test based in the Kolmogorov-Smirnov goodness-of-fit test. 9th Data Management Workshop of EUMETNET, El Escorial (Madrid), 6th-8th November.

Monjo, R.; Martin-Vide, J. (2016). Daily precipitation concentration around the world according to several indices. International Journal of Climatology. DOI: 10,1002/ joc. 4596 .

Monjo, R. (2016). Measure of rainfall time structure using the dimensionless n-index. Climate Research, 67, 71-86. doi: http://dx.doi.org/10,3354/cr01359.

Olsson, J.; Niemczynowicz, J.; Berndtsson, R.; Larson, M. (1992), An analysis of the rainfall time structure by box counting-some practical implications. J Hydrol 137, 261-277.

Taouti, M.B; Chettih, M. (2014). Fractal and multifractal analyses of the temporal structure of daily rainfall in a Mediterranean climate in northern Algeria. Tethys 11, 3-12. doi: http://dx.doi.org/10,3369/tethys.2014.11.01 